\title{
Localizing the L5 Vertebra Using Nerve Morphology on MRI: An Accurate and Reliable Technique
}

\author{
(D) M.E. Peckham, (D)T.A. Hutchins, (D) S.E. Stilwill, (D) M.K. Mills, DB.J. Morrissey, DE.A.R. Joiner, DR.K. Sanders, (D) G.J. Stoddard, and
} (iD).M. Shah

\begin{abstract}
BACKGROUND AND PURPOSE: Multiple methods have been used to determine the lumbar vertebral level on MR imaging, particularly when full spine imaging is unavailable. Because postmortem studies show $95 \%$ accuracy of numbering the lumbar vertebral bodies by counting the lumbar nerve roots, attention to lumbar nerve morphology on axial MR imaging can provide numbering clues. We sought to determine whether the L5 vertebra could be accurately localized by using nerve morphology on MR imaging.
\end{abstract}

MATERIALS AND METHODS: One hundred eight cases with full spine MR imaging were numbered from the C2 vertebral body to the sacrum with note of thoracolumbar and lumbosacral transitional states. The origin level of the L5 nerve and iliolumbar ligament were documented in all cases. The reference standard of numbering by full spine imaging was compared with the nerve morphology numbering method. Five blinded raters evaluated all lumbar MRIs with nerve morphology technique twice. Prevalence and bias-adjusted $\kappa$ were used to measure interrater and intrarater reliability.

RESULTS: The L5 nerve arose from the 24th presacral vertebra (L5) in 106/108 cases. The percentage of perfect agreement with the reference standard was $98.1 \%$ (95\% Cl, 93.5\%-99.8\%), which was preserved in transitional and numeric variation states. The iliolumbar ligament localization method showed $83.3 \%$ (95\% Cl, 74.9\%-89.8\%) perfect agreement with the reference standard. Inter- and intrarater reliability when using the nerve morphology method was strong.

CONCLUSIONS: The exiting L5 nerve can allow accurate localization of the corresponding vertebrae, which is essential for preprocedure planning in cases where full spine imaging is not available. This neuroanatomic method displays higher agreement with the reference standard compared with previously described methods, with strong inter- and intrarater reliability.

ABBREVIATIONS: LSTV = lumbosacral transitional vertebrae; PABAK = prevalence-adjusted bias-adjusted $\kappa ;$ PSV $=$ presacral vertebrae; VNV $=$ vertebral numeric variation

A ccurate and reliable spine numbering is important for the diagnosis of pathology and preprocedure planning. This can be challenging in patients with vertebral numeric variation (VNV) or lumbosacral transitional vertebrae (LSTV), particularly when full spine imaging is unavailable. VNV refers to the variation of the total number of presacral vertebrae (PSV). Approximately $89 \%$ of the population have 24 PSV (5 lumbar-type verte-

Received March 10, 2017; accepted after revision May 23

From the Neuroradiology Division (M.E.P., T.A.H., G.J.S., L.M.S.) and Musculoskeletal Division (S.E.S., M.K.M., R.K.S.), Departments of Radiology and Imaging Sciences (B.J.M., E.A.R.J.), University of Utah Health Sciences Center, Salt Lake City, Utah. Paper previously presented at the American Society of Spine Radiology Annual Symposium, February 23-26, 2017; San Diego, California. (Awarded 1st place in the Mentor Award category.)

Please address correspondence to Miriam E. Peckham, MD, Neuroradiology Division, Departments of Radiology and Imaging Sciences, University of Utah Health Sciences Center, 30 North, 1900 East, \#1A071, Salt Lake City, UT 84132; e-mail: Miriam.Peckham@hsc.utah.edu; @Miriam_Peckham

http://dx.doi.org/10.3174/ajnr.A5311 brae), $8 \%$ have 25 PSV (6 lumbar-type vertebrae), and 3\% have 23 PSV (4 lumbar-type vertebrae). ${ }^{1}$ LSTV are congenital spinal anomalies in which an elongated transverse process of the last lumbar vertebra fuses with the "first" sacral segment to varying degrees. ${ }^{2}$ The morphologic variation of LSTV can range from partial/complete L5 sacralization to partial/complete S1 lumbarization. ${ }^{3,4}$ The prevalence of LSTV in the population varies throughout the literature because of differences in definition and diagnostic modalities. ${ }^{1,4-6}$ LSTV can also vary with sex, with lumbarization of $\mathrm{S} 1$ seen more commonly in women and sacralization found to be more common in men. ${ }^{3}$ A person can have VNV without LSTV, or conversely, one can have LSTV without VNV. ${ }^{1}$ Approximately $5 \%$ of subjects have been found to have both. ${ }^{1}$

Multiple anatomic landmarks have been used to determine the lumbar vertebral level in cases without full spine imaging. A leading method of localizing the iliolumbar ligament, most frequently arising from L5, has been found less accurate in the setting of 
LSTV and VNV. ${ }^{7-11}$ Other landmarks, including the level of the conus, right renal artery, superior mesenteric artery, aortic bifurcation, and iliac crest height, are also less accurate. ${ }^{9,12-14}$ Choosing the appropriate level for surgical or interventional procedures is essential and relies on accurately and reliably numbering the spine in patients with "normal" anatomy as well as those with variant or transitional anatomy. ${ }^{4,15}$ This is especially important in patients with LSTV and/or VNV undergoing surgical planning, as up to $32 \%$ of neurosurgeons have reported an event of wronglevel spinal surgery occurring at least once in their careers. ${ }^{16}$ LSTV can also create challenges for approach in interventional pain procedures and can increase the risk of iatrogenic vascular injury. ${ }^{17}$

Multiple imaging modalities have been used to evaluate LSTV and VNV, with MR imaging found to be most reliable. ${ }^{18}$ Anteroposterior radiographs have demonstrated high intermodality agreement with MR imaging. ${ }^{19}$ Studies show that one can accurately number the vertebrae by counting down from $\mathrm{C} 2$ to the sacrum on sagittal MR imaging by using a cross-referencing tool. ${ }^{1,8,19,20}$ Although most counting methods have focused on the ossified structures, 1 postmortem study numbered the vertebrae by dorsal spinal nerve morphology and found up to $95 \%$ probability that the lower spinal nerves correspond to their respective spinal segment. ${ }^{21}$ We hypothesized that nerve morphology on lumbar spine MR imaging would aid in L5 vertebra localization, particularly when full spine imaging was not available. We aimed 1) to determine whether MR imaging morphologic features of the lumbar nerves could be used to distinguish the lower lumbar levels and 2) to apply these characteristics in localizing the L5 vertebra.

\section{MATERIALS AND METHODS}

This retrospective study, performed over 7 months, was approved by the institutional review board and investigators were compliant with the Health Insurance Portability and Accountability Act.

\section{Patients}

We searched our picture archiving and communication system for patients aged 18 years and older who had MR imaging of the full spine and radiographic imaging (CT or radiographs) of the thoracolumbar and lumbosacral junctions within the last 4 years (2013-2016). Patients without these studies were excluded. Patients with congenital vertebral segmentation anomalies were also excluded because of the possibility of associated nerve anomalies. The indications for most of these studies were back pain and metastatic disease, and patients were included if the osseous structures and nerves could be delineated.

\section{Vertebral Body Count}

Two investigators, a neuroradiology faculty member (L.M.S.) with more than 10 years' experience in spine imaging and a neuroradiology fellow (M.E.P.), reviewed each case and documented the total number of presacral vertebrae by counting down from C2 to the sacrum on MR imaging. Radiographic images of the thoracic and lumbar spine were reviewed to document rib count as well as evaluate transitional anatomy at the thoracolumbar and lumbosacral junctions. O'Driscoll staging ${ }^{22}$ and the Castellvi method $^{23}$ were used to classify the lumbosacral anatomy. The

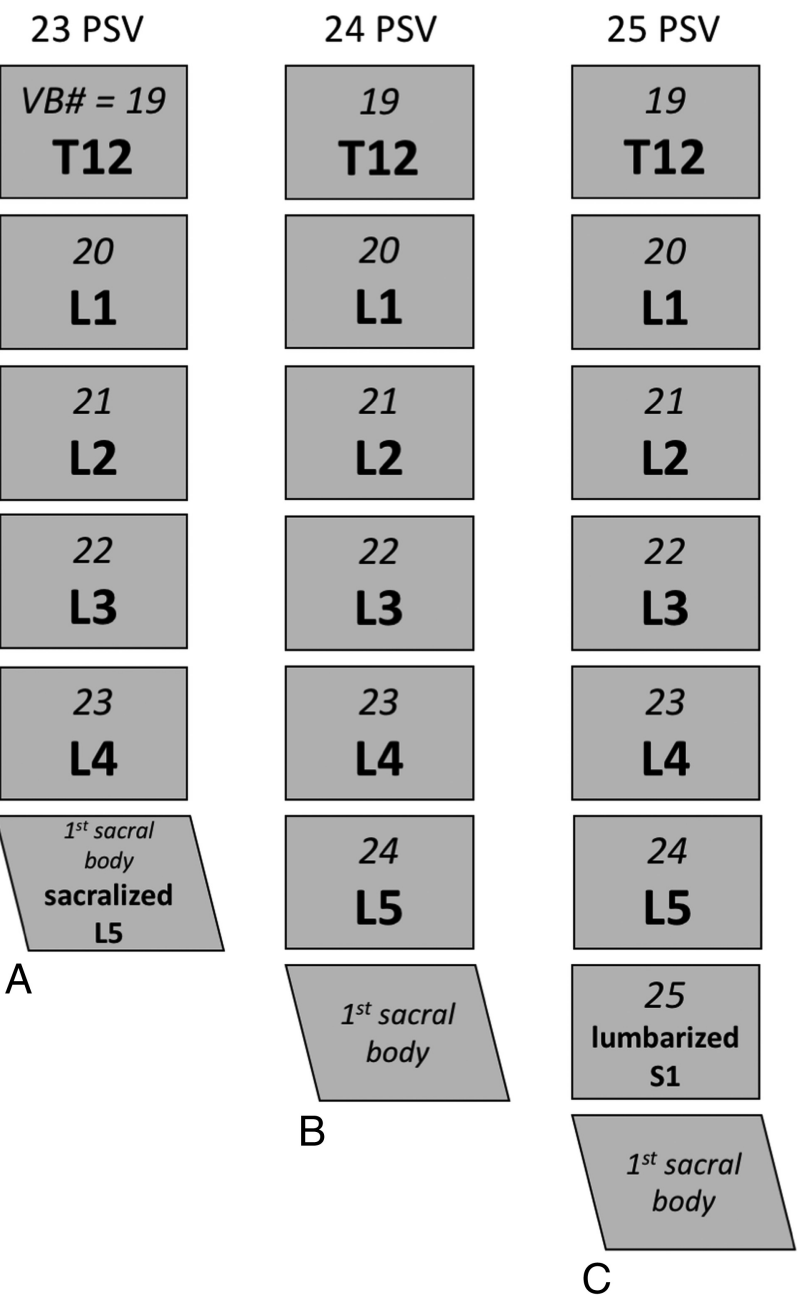

FIG 1. Graphic demonstrating our method for vertebral body numbering. When counting down from $\mathrm{C} 2$, patients with only 4 lumbar-type vertebral bodies (sacralized L5) have 23 PSV (A), patients with 5 lumbar-type vertebral bodies have 24 PSV (B), and patients with 6 lumbar-type vertebral bodies (lumbarized S1) have $25 \operatorname{PSV}(C)$.

level of the iliolumbar ligament and L5 nerve were also documented in all cases.

Vertebral numbering was performed as follows: the first 7 vertebrae were considered cervical, and the next 12 vertebrae were considered to be thoracic even in cases with an anomalous number of ribs. ${ }^{1}$ In the cases with 13 rib-bearing vertebrae, we considered it "lumbar thoracization" with L1 having supranumery ribs. After T12, the vertebrae were counted as lumbar-type, extending to the level of the lumbosacral junction. Based on morphology and laterality per the Castellvi classification, ${ }^{23}$ if the lower lumbar transverse processes had either unilateral or bilateral nonfused articulations with the sacrum (partial L5 sacralization), they were classified as either Castellvi 1 or 2. If the transverse processes were either unilaterally or bilaterally fused to the sacrum (complete L5 sacralization), the LSTV were classified as either Castellvi 3 or 4. The total number of PSV was the sum of cervical, thoracic, and lumbar segments. The 24th vertebra was considered L5 in all cases, even in those with VNV or LSTV (Fig 1). In LSTV cases, a patient with partial L5 sacralization (unilateral or bilateral assimilation joints without osseous fusion) was considered to have 24 


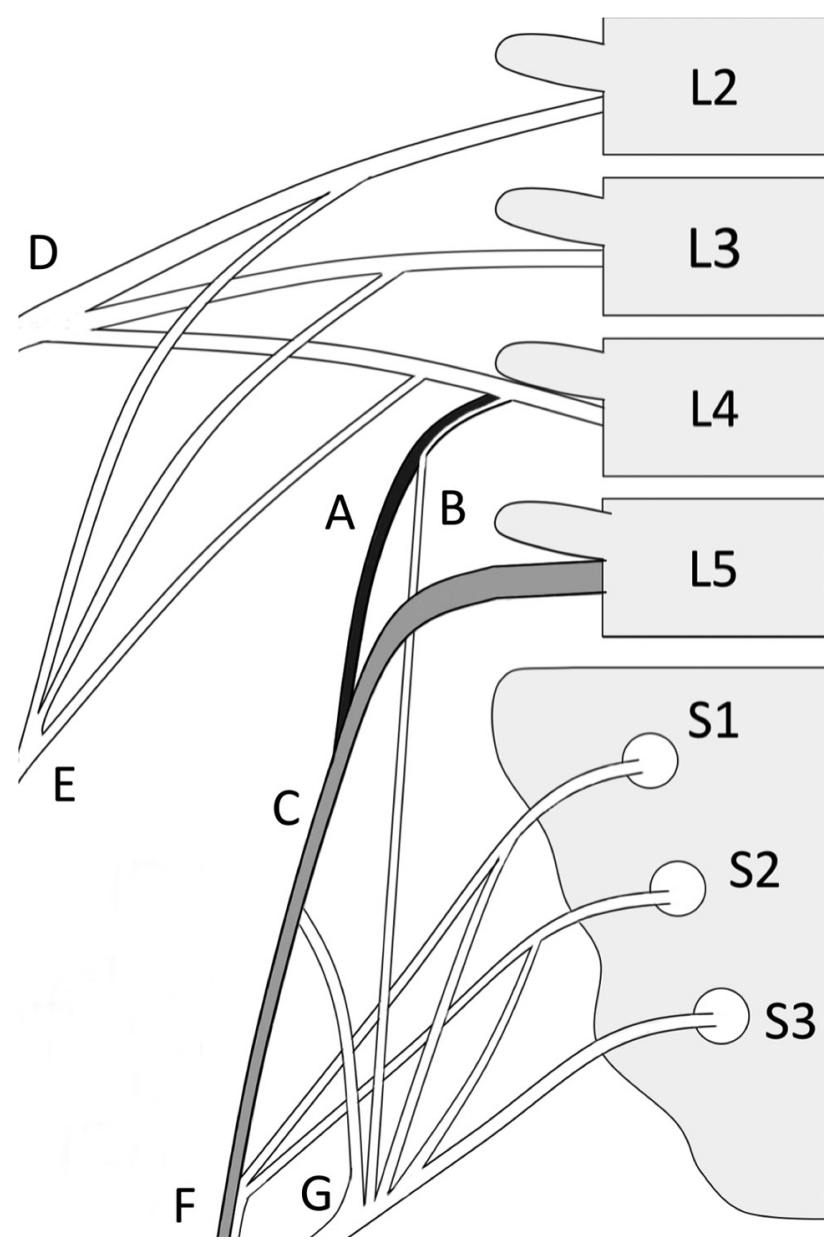

FIG 2. Schematic demonstrating the divisions of the lumbosacral plexus. The $L 4$ nerve divides soon after exiting the neural foramen into peroneal (black) (A) and tibial (B) components, with the peroneal component joining the lateral fibers of $L 5$ (gray) (C). The $L 4$ nerve also contributes to both the femoral (D) and obturator (E) nerves. L5 is the only lumbar nerve that does not have a proximal division. Branches of L4-S2 make up the common peroneal nerve (F), and branches of L4-S3 make up the tibial nerve (G), which together comprise components of the sciatic nerve (not illustrated). The MRI morphology of the L4 peroneal component and L5 nerve are of special importance for localization; thus, they are shaded in this figure.

PSV, whereas a patient with complete L5 sacralization (unilateral or bilateral assimilation joints with osseous fusion) was considered to have 23 PSV. By the same Castellvi classification method, in those patients with lumbarization of S1, the patient was considered to have 24 PSV when S1 was partially lumbarized and 25 PSV if $S 1$ was completely lumbarized. ${ }^{1,23}$

\section{L5 Nerve Localization}

The L5 nerve was identified by using 3 anatomic characteristics. First, L5 is typically the only lumbar nerve that does not split proximally and was identified on MR imaging by its nonsplitting course (Fig 2). Second, the insertion of the L4 peroneal branch along the lateral aspect of the L5 nerve, commonly seen at the level of the sacrum in patients with normal anatomy, was a helpful characteristic (Fig 3). Finally, the caliber of nerves along the sacrum aided in localization; specifically, the nonsplitting L5 nerve was approximately twice the caliber of the L4 peroneal branch at the level of the sacrum. This sign was particularly helpful in thin-

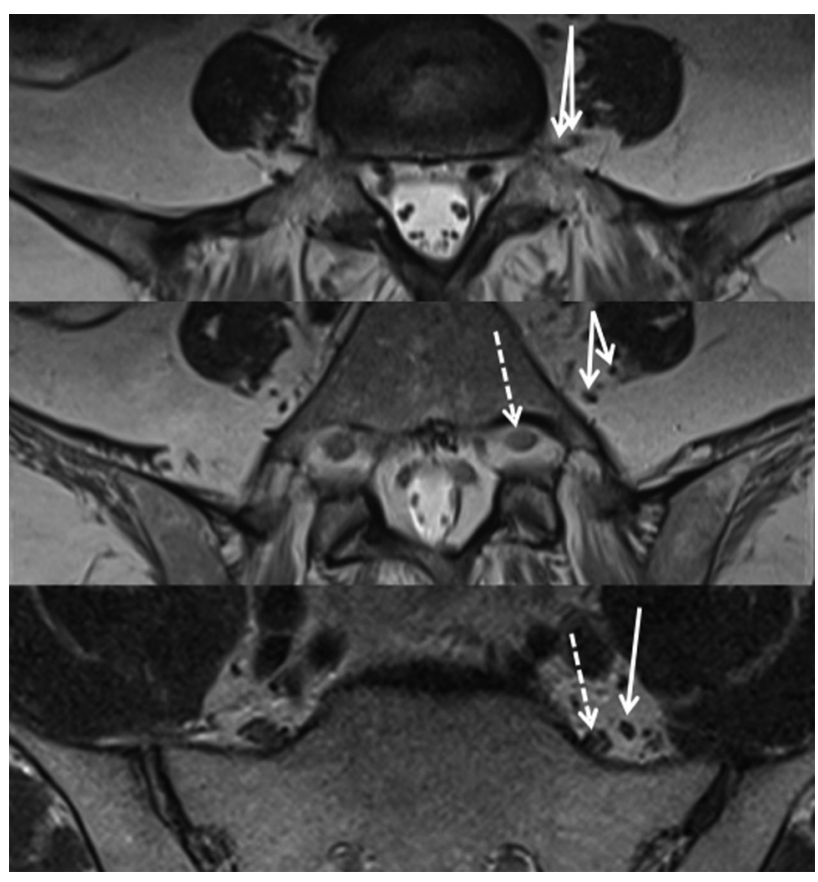

FIG 3. Consecutive cranial to caudal axial T2-weighted MR images demonstrate L4 and L5 nerve root anatomy. The L4 nerve root splits proximally into tibial and peroneal branches (solid arrows). The peroneal branch extends caudally and joins with the $L 5$ nerve root (dashed arrow) along its anterolateral aspect at the level of the lateral sacrum.

ner patients, in whom the psoas muscle obscured the exiting L4 nerve (Fig 4).

\section{Interrater and Intrarater Reliability}

Five blinded raters of various stages of training, including 2 residents (2nd year and 4th year), 1 junior faculty member (1 year postfellowship), and 2 senior faculty members ( 5 and 7 years postfellowship) from both neuroradiology and musculoskeletal radiology subspecialties reviewed all 108 MR imaging lumbar spines in random order on 2 occasions, separated by 2 months. Before reviewing the cases, the raters were given a brief tutorial on lumbosacral plexus anatomy, MR imaging nerve appearance, and the method of nerve morphology numbering. Each rater was asked to localize the L5 nerve on lumbar spine MR imaging and determine normal (5 lumbar-type vertebral bodies) or LSTV anatomy (ie, lumbarized S1 or sacralized L5) by using the nerve morphology method and lumbosacral osseous anatomy. No other imaging was provided. Those results were compared with the reference standard as determined by full spine MR imaging.

\section{Statistical Analyses}

Patient sample size was determined by the rate of variant anatomy in the population with more than 100 patients chosen to achieve a 95\% CI. Descriptive statistics were calculated for PSV. To verify the reliability of the nerve morphology method for denoting L5, we determined at which spinal level the L5 nerve exited and expressed this as a percentage agreement with the reference standard labeling. Although the $\kappa$ coefficient is more widely familiar, it has an anomaly when data are clumped into 1 cell of the cross-tabulation table between raters. Therefore, the more relevant and ap- 


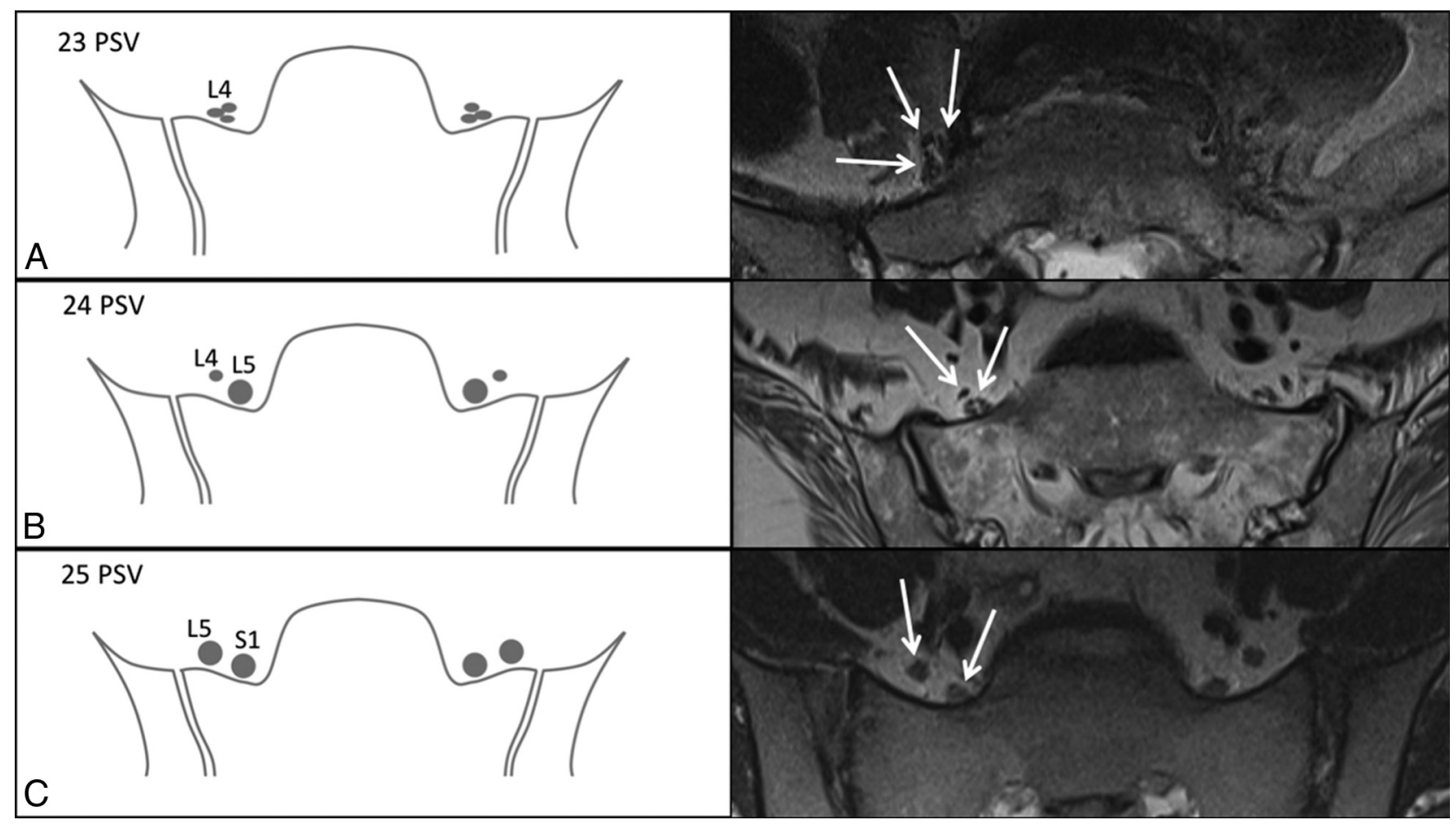

FIG 4. Axial T2-weighted MR images at the level of the sacrum with corresponding graphics demonstrating how the caliber of the nerve roots along the sacrum can be used to identify the number of lumbar vertebral segments. In patients with 4 lumbar segments, the L4 nerve root is seen splitting over the lateral sacrum ( $A$, arrows). In patients with 5 lumbar segments, the peroneal branch of $L 4$ joins the $L 5$ nerve root, which is twice the caliber of $L 4$ ( $B$, arrows). In patients with more than 5 lumbar segments, 2 nerves of similar caliber will be seen along the lateral sacral wing, representing L5 laterally and S1 medially (C, arrows).

propriate prevalence-adjusted bias-adjusted $\kappa$, or PABAK, was used to measure interrater and intrarater reliability, which gives the true proportion of agreement beyond chance agreement regardless of unbalanced data patterns. ${ }^{24}$ Although a formula for computing the PABAK interreliability for more than 2 raters simultaneously is not available, using the mean PABAK and range of confidence limits provides a reasonable approximation of the interrater reliability of the 5 raters simultaneously. Statistical analyses were performed by using commercial statistical analysis software (STATA Statistical Software: Release 14; StataCorp, College Station, Texas).

\section{RESULTS}

One hundred eight patients were randomly selected from this data base inquiry (60 females). The combined subject group ranged in age from $18-90$ years (mean, 51.9 years \pm 16.9 ). The female patients ranged in age from 18-90 (mean, 50.1 years), and the male patients ranged in age from 29-87 (mean, 54.1 years).

\section{Vertebral Body Count}

Sixteen of 108 patients had VNV (14.8\%), 7 of whom had 23 PSV (6.5\%) and 9 of whom had 25 PSV (8.3\%). Ninety-two patients had 24 PSV (86\%). Thirty of 108 patients had LSTV (29.7\%) with 24 of these patients having Castellvi type 1 or 2 and 6 having Castellvi type $3 \mathrm{a}$ or $3 \mathrm{~b}$. None of the patients had Castellvi type 4. Nine of 16 patients with VNV also had LSTV.

Twelve patients had hypoplastic ribs at T12, 8 of whom also had LSTV. In addition, 6 patients had 13 rib-bearing vertebral bodies, and none of these patients had LSTV. One patient had only 11 rib-bearing thoracic vertebrae and 6 non-rib-bearing bodies (total of $24 \mathrm{PSV}$ ) with partial sacralization of L5. One patient had bilateral cervical ribs at C7.

\section{L5 Nerve Localization}

The L5 nerve was identified in all patients and arose from the 24th PSV (L5) in 106/108 cases. The percentage of perfect agreement with the reference standard was $98.1 \%$ (95\% CI, 93.5\%-99.8\%). This agreement was preserved in cases with LSTV and VNV. In the 2 cases that were incongruous with the reference standard, the L5 nerve arose from a lumbarized S1 vertebra, and in both of these cases, there was variant thoracolumbar anatomy with supranumery ribs at L1. The percentage of perfect agreement with the reference standard when using the iliolumbar ligament localization method was $83.3 \%$ (95\% CI, 74.9\%-89.8\%), accurately identifying the level L5 in 90/108 cases. In the cases of nonagreement, either the iliolumbar ligament did not arise from the 24th PSV, arose from 2 different levels, accessory ligaments were present, or the ligaments were difficult to identify.

\section{Interrater and Intrarater Reliability}

Computing PABAK for all possible pairs of comparisons of interrater reliability yielded a range of $0.83-0.96$. The average PABAK was excellent at 0.89 (Table 1). The interrater reliability between each rater, and the reference standard are reported in Table 2 . The intrarater reliability comparing a rater's scores on 2 separate occasions is reported in Table 3. 
Table 1: PABAK interrater reliability coefficients between each pair of raters with $95 \% \mathrm{Cl}^{\mathrm{a}}$

\begin{tabular}{lcccc}
\hline Rater & $\mathbf{2}$ & $\mathbf{3}$ & $\mathbf{4}$ & $\mathbf{5}$ \\
\hline 1 & $0.91(0.83-0.99)$ & $0.96(0.91-1.0)$ & $0.91(0.83-0.99)$ & $0.89(0.80-0.98)$ \\
2 & & $0.94(0.88-1.0)$ & $0.85(0.75-0.95)$ & $0.83(0.73-0.94)$ \\
3 & & & $0.91(0.83-0.99)$ & $0.85(0.75-0.95)$ \\
4 & & & & $0.83(0.73-0.94)$ \\
\hline
\end{tabular}

${ }^{a}$ Interrater reliabilities between each pair of raters ranged from $0.83-0.96$. The average coefficient was 0.89 . The smallest $95 \% \mathrm{Cl}$ lower limit was 0.73 , and the largest upper limit was 1.0. Although a formula for computing the PABAK interreliability for more than 2 raters simultaneously is not available, using this mean and range of confidence limits provides a reasonable approximation of the interrater reliability of the 5 raters simultaneously (PABAK, 0.89; $95 \% \mathrm{Cl}, 0.73-1.0$ ).

$\begin{aligned} & \text { Table 2: Interrater reliability between each rater and the } \\
& \text { reference standard }\end{aligned}$
\begin{tabular}{lc} 
Rater & PABAK Coefficient $(95 \% \mathrm{CI})$ \\
\hline 1 & $0.91(0.83-0.99)$ \\
2 & $0.81(0.70-0.92)$ \\
3 & $0.87(0.78-0.96)$ \\
4 & $0.85(0.75-0.95)$ \\
5 & $0.94(0.88-1.0)$ \\
\hline
\end{tabular}

Table 3: Intrarater reliability comparing each rater's scoring on 2 separate occasions

\begin{tabular}{lc}
\hline Rater & PABAK Coefficient $(95 \% \mathrm{CI})$ \\
\hline 1 & $0.92(0.85-0.99)$ \\
2 & $0.85(0.75-0.95)$ \\
3 & $0.91(0.83-0.99)$ \\
4 & $0.89(0.80-0.98)$ \\
5 & $0.78(0.65-0.89)$ \\
\hline
\end{tabular}

\section{DISCUSSION}

Developing an accurate and reliable method for numbering the lumbar vertebrae when complete spine imaging is not available has been difficult, especially in patients with LSTV and VNV. We found that the neuroanatomic MR imaging features of the exiting L5 nerve can allow accurate localization of the L5 vertebra.

Embryologically, the neural structures arise from the ectoderm, whereas the osseous scaffold arises from the mesoderm. The notochord is central to the development of the spine, acting as a frame for organization of the mesodermal cells from which eventually arises the vertebral column. Signal from the notochord and neural tube during the sixth week leads to chondrification and ultimately ossification. ${ }^{25,26}$ The cervical spinal segments demonstrate morphologic stability with a fixed number of 7 vertebrae, whereas the thoracic and lumbar segments can vary. ${ }^{27-29}$ An association of transitional thoracolumbar junction anatomy with concomitant LSTV has been noted. ${ }^{7} \mathrm{Al}-$ though the osseous structures show variation in up to $16 \%$ of the population, the neural structures have been shown to have less variability. $1,6,21,25,26$

The L5 nerve can be localized on MR imaging by using the morphologic features of the lumbosacral plexus. First, L5 is typically the only lumbar nerve without proximal branching. The L1-L4 nerves all split proximally just after exiting the neural foramen. The "normal" L4 nerve contributes to the femoral and obturator nerves. A posterior fascicle of $\mathrm{L} 4$ joins the lateral surface of L5 proximally, eventually making up the lateral/peroneal part of the sciatic nerve. This L4 contribution to the peroneal component of the sciatic nerve is small (Fig 2). Along with L4, the L5-S2 nerves contribute to the common peroneal and tibial components of the sciatic nerve. One can follow the first "nonsplitting" nerve to determine the level of the L5 vertebral body. For example, if the first nonsplitting nerve is tracked back to the first sacral body, it supports the patient only having 4 lumbar-type vertebrae with sacralization of L5 (23 PSV) (Fig 1A). If the first nonsplitting nerve is tracked back to a vertebral body 2 levels above the first sacral body, it supports the patient having more than 5 lumbartype vertebrae (lumbarization of S1, 25 PSV) (Fig 1C). Second, the L4 peroneal branch inserts along the lateral aspect of the L5 nerve, commonly at the level of the sacrum in patients with nonvariant anatomy. Third, the caliber of nerves along the sacrum can aid with localization; that is, the nonsplitting L5 nerve is approximately twice the size of the L4 peroneal branch at the level of the sacrum. Differences in nerve caliber along the sacrum can be useful for localization in patients with a paucity of abdominal fat where the psoas muscle obscures L4 and when there are confounding adjacent small vascular structures. In patients with 23 PSV, the larger caliber L5 nerve arises from the first sacralized foramen, and the L4 nerve divides along the lateral sacrum (Fig $4 A$ ). In patients with $24 \mathrm{PSV}$, both the peroneal branch of $\mathrm{L} 4$ and the L5 nerve are present along the lateral sacrum, with L5 approximately twice the caliber of the L4 peroneal branch (Fig 4B). In patients with 25 PSV, the nerves coursing along the sacrum will be of similar caliber as they represent the L5 and S1 nerve roots (Fig $4 C$ ). Given that the nerves can vary in size such that L5 may not be equal in size to S1 in all cases but slightly smaller, caliber should not be used in isolation of the other morphologic characteristics.

Assessment of nerve morphology can be challenging in patients with severe neural foraminal narrowing and facet disease, which obscure evaluation of the proximal nerves, and when there is pathology deforming the nerve (eg, peripheral nerve sheath tumors or chronic inflammatory demyelinating polyneuropathy). Patients with congenital vertebral segmentation anomalies (eg, hemivertebrae) also present a numbering challenge because there may be concomitant variant lumbosacral plexus anatomy (ie, duplicated nerves). An additional potential pitfall includes when the patient's L4 peroneal branch is borderline in caliber, not distinctly $<50 \%$ the size of L5 along the lateral sacrum. In these cases, one should follow the nerves proximally to determine whether 1 of the nerves divides; otherwise, additional studies (eg, CT chest and abdomen) may be helpful for vertebral body counting. This nerve morphology method works best with sequential axial images so that the nerves can be tracked to the exiting neural foramen. Different types of conjoined nerve roots may pose another numbering challenge, albeit less common. ${ }^{30}$ As is advocated by most radiologists, the imaging report should state how the vertebral bodies were numbered and if there is transitional or variant anatomy to avoid confusion for the referring clinician.

The 2 cases where the nerve morphology method was discordant with the reference standard demonstrated nerves with L5 morphology arising from a lumbarized S1 in patients with 25 PSV. The L 5 nerves split proximally, which made them more consistent with L4 morphology. In both cases, there was "lumbar thoracization" with 13 rib-bearing vertebrae without LSTV. Although the 4 other patients with 13 rib-bearing vertebrae followed the expected nerve morphology, we highlight the importance of being aware of altered lumbosacral nerve distribution in the set- 
ting of transitional thoracolumbar anatomy. We posit that in patients with transitional thoracolumbar anatomy and 25 PSV, the L5 nerve assumes "the role of the L4 nerve," providing the peroneal component of the forming sciatic nerve trunk, whereas the sciatic nerve trunk is made up predominantly by the S1 nerve root. $^{31}$

Alternatively, there may be "thoracic lumbarization" in which there are 11 rib-bearing vertebral bodies and 6 non-rib-bearing bodies. In this situation, the L4 nerve may contribute the peroneal component, and the L5 nerve may contribute the tibial component to the forming sciatic nerve at 1 spinal level higher than in normal anatomy. This may not be revealing in the nerve morphology, as in our 1 patient with 11 rib-bearing vertebrae and 6 non-rib-bearing vertebrae (total of $24 \mathrm{PSV}$ ). A low number of the population have thoracolumbar transitional anatomy, reflecting why this nerve morphology technique works the majority of the time ( $95 \%$ by postmortem studies). Although using nerve morphology is not a perfect technique, it does enable lumbar spine numbering to be rapidly deduced on MR imaging and quickly provides clues for when further evaluation with vertebral counting is warranted.

The nerve method is based on the morphologic characteristics of the exiting spinal nerves; however, some studies suggest that there are some variations in the "physiologic" nerve. Intraoperative electrophysiologic monitoring of evoked electromyography in patients with 24 PSV compared with 25 PSV showed that the "L6" nerve was equivalent to the S1 nerve root not only morphologically, but also physiologically as it innervated the biceps femoris. ${ }^{32}$ Seyfert ${ }^{33}$ used the cremasteric reflex in 50 male patients and correlated it to spine imaging. He found that the lumbosacral dermatome lies more ventrally in patients with a cranial displacement of the thoracolumbar or lumbosacral vertebral transition, which may reflect the variant plexus position. Kim et $\mathrm{al}^{34}$ performed selective nerve root blocks by using electrical stimulation in patients having transitional vertebrae with lumbosacral radiculopathy. They found that the distribution of motor and sensory symptoms caused by the lumbarized S1 (L6) nerve root stimulation was similar to that of the S1 nerve root stimulation in the normal configuration, whereas the distribution of motor and sensory symptoms caused by the sacralized L4 nerve root stimulation was similar to that of L5 nerve root stimulation in the normal configuration.

The high interrater reliability and overall strong intrarater reliability of this method shows that it can be realistically implemented across subspecialties and is reproducible in the hands of users. Familiarity of the lumbosacral plexus anatomy is easily attained by the practicing radiologist and facilitates application of this neuroanatomic method of spine numbering. There is variability in how practitioners number the spine, without taking into account LSTV and VNV. We believe this nerve method will provide consistency in reporting between readers. Using nerve morphology can also aid in anatomic localization for symptoms that follow specific nerve distributions. This can be useful both in diagnostic studies and interventional spine procedures and is especially helpful in patients with variant anatomy. Localizing the L5 nerve is the key for preprocedural planning and typically only the osseous structures are used as preprocedural/procedural assess- ment modalities (eg, radiographs and fluoroscopy). Identifying the L5 nerve and determining the corresponding vertebral level will allow appropriate localization during procedures.

\section{CONCLUSIONS}

The level of the exiting L5 nerve can allow accurate localization of the corresponding vertebrae, particularly when full spine imaging is not available. This neuroanatomic method displays higher agreement with the reference standard compared with previously described methods. The strong inter- and intrarater reliability illustrates that this method can provide consistency in reporting between readers and is essential for accuracy in preprocedure planning.

\section{REFERENCES}

1. Paik NC, Lim CS, Jang HS. Numeric and morphological verification of lumbosacral segments in $\mathbf{8 2 8 0}$ consecutive patients. Spine 2013; 38:E573-78 CrossRef Medline

2. Hughes RJ, Saifuddin A. Imaging of lumbosacral transitional vertebrae. Clin Radiol 2004;59:984-91 CrossRef Medline

3. Mahato NK. Relationship of sacral articular surfaces and gender with occurrence of lumbosacral transitional vertebrae. Spine J 2011; 11:961-65 CrossRef Medline

4. Konin GP, Walz DM. Lumbosacral transitional vertebrae: classification, imaging findings, and clinical relevance. AJNR Am J Neuroradiol 2010;31:1778-86 CrossRef Medline

5. Apazidis A, Ricart PA, Diefenbach CM, et al. The prevalence of transitional vertebrae in the lumbar spine. Spine J 2011;11:858-62 CrossRef Medline

6. Tang M, Yang XF, Yang SW, et al. Lumbosacral transitional vertebra in a population-based study of $\mathbf{5 8 6 0}$ individuals: prevalence and relationship to low back pain. Eur J Radiol 2014;83:1679-82 CrossRef Medline

7. Carrino JA, Campbell PD Jr, Lin DC, et al. Effect of spinal segment variants on numbering vertebral levels at lumbar MR imaging. $R a-$ diology 2011;259:196-202 CrossRef Medline

8. Bressler EL. Numbering of lumbosacral transitional vertebrae on MRI. AJR Am J Roentgenol 2007;188:W210; author reply W211 CrossRef Medline

9. Tureli D, Ekinci G, Baltacioglu F. Is any landmark reliable in vertebral enumeration? A study of 3.0-Tesla lumbar MRI comparing skeletal, neural, and vascular markers. Clin Imaging 2014;38:792-96 CrossRef Medline

10. Farshad-Amacker NA, Lurie B, Herzog RJ, et al. Is the iliolumbar ligament a reliable identifier of the $\mathrm{L} 5$ vertebra in lumbosacral transitional anomalies? Eur Radiol 2014;24:2623-30 CrossRef Medline

11. Hughes RJ, Saifuddin A. Numbering of lumbosacral transitional vertebrae on MRI: role of the iliolumbar ligaments. AJR Am J Roentgenol 2006;187:W59-65 CrossRef Medline

12. Farshad-Amacker NA, Aichmair A, Herzog RJ, et al. Merits of different anatomical landmarks for correct numbering of the lumbar vertebrae in lumbosacral transitional anomalies. Eur Spine J 2015; 24:600-08 CrossRef Medline

13. Tokgoz N, Ucar M, Erdogan AB, et al. Are spinal or paraspinal anatomic markers helpful for vertebral numbering and diagnosing lumbosacral transitional vertebrae? Korean J Radiol 2014;15: 258-66 CrossRef Medline

14. Lee $\mathrm{CH}$, Seo BK, Choi YC, et al. Using MRI to evaluate anatomic significance of aortic bifurcation, right renal artery, and conus medullaris when locating lumbar vertebral segments. AJR Am J Roentgenol 2004;182:1295-300 CrossRef Medline

15. Crawford CH 3rd, Glassman SD, Gum JL, et al. Conflicting calculations of pelvic incidence and pelvic tilt secondary to transitional lumbosacral anatomy (lumbarization of S-1): case report. J Neurosurg Spine 2017;26:45-49 CrossRef Medline 
16. Jhawar BS, Mitsis D, Duggal N. Wrong-sided and wrong-level neurosurgery: a national survey. J Neurosurg Spine 2007;7:467-72 CrossRef Medline

17. Josiah DT, Boo S, Tarabishy A, et al. Anatomical differences in patients with lumbosacral transitional vertebrae and implications for minimally invasive spine surgery. J Neurosurg Spine 2017;26:137-43 CrossRef Medline

18. Farshad-Amacker NA, Lurie B, Herzog RJ, et al. Interreader and intermodality reliability of standard anteroposterior radiograph and magnetic resonance imaging in detection and classification of lumbosacral transitional vertebra. Spine J 2014;14:1470-75 CrossRef Medline

19. Paik NC, Lim CS, Jang HS. Numbering of vertebrae on MRI using a PACS cross-referencing tool. Acta Radiol 2012;53:785-89 CrossRef Medline

20. Shabshin N, Schweitzer ME, Carrino JA. Anatomical landmarks and skin markers are not reliable for accurate labeling of thoracic vertebrae on MRI. Acta Radiol 2010;51:1038-42 CrossRef Medline

21. Prat D, Gagnard G, Cousineau J. Numbering of the dorsal spinal nerve roots in man from the 12th thoracic nerve to the coccygeal nerve [Article in French]. Bull Assoc Anat (Nancy) 1983;67:331-36 Medline

22. O'Driscoll CM, Irwin A, Saifuddin A. Variations in morphology of the lumbosacral junction on sagittal MRI: correlation with plain radiography. Skeletal Radiol 1996;25:225-30 CrossRef Medline

23. Castellvi AE, Goldstein LA, Chan DP. Lumbosacral transitional vertebrae and their relationship with lumbar extradural defects. Spine (Phila Pa 1976) 1984;9:493-95 CrossRef

24. Byrt T, Bishop J, Carlin JB. Bias, prevalence and kappa. J Clin Epidemiol 1993;46:423-29 CrossRef Medline
25. Scaal M. Early development of the vertebral column. Semin Cell Dev Biol 2016;49:83-91 CrossRef Medline

26. Thawait GK, Chhabra A, Carrino JA. Spine segmentation and enumeration and normal variants. Radiol Clin North Am 2012;50: 587-98 CrossRef Medline

27. Galis F. Why do almost all mammals have seven cervical vertebrae? Developmental constraints, Hox genes, and cancer. J Exp Zool 1999; 285:19-26 CrossRef Medline

28. Narita Y, Kuratani S. Evolution of the vertebral formulae in mammals: a perspective on developmental constraints. J Exp Zool B Mol Dev Evol 2005;304:91-106 CrossRef Medline

29. Wigh RE. Classification of the human vertebral column: phylogenetic departures and junctional anomalies. Med Radiogr Photogr 1980;56:2-11 Medline

30. Postacchini F, Urso S, Ferro L. Lumbosacral nerve-root anomalies. J Bone Joint Surg Am 1982;64:721-29 CrossRef Medline

31. Chang HS, Nakagawa $H$. Altered function of lumbar nerve roots in patients with transitional lumbosacral vertebrae. Spine (Phila $\mathrm{Pa}$ 1976) 2004;29:1632-35; discussion 1635 CrossRef Medline

32. Hinterdorfer P, Parsaei B, Stieglbauer K, et al. Segmental innervation in lumbosacral transitional vertebrae (LSTV): a comparative clinical and intraoperative EMG study. J Neurol Neurosurg Psychiatry 2010;81:734-41 CrossRef Medline

33. Seyfert $S$. Dermatome variations in patients with transitional vertebrae. J Neurol Neurosurg Psychiatry 1997;63:801-03 CrossRef

34. Kim YH, Lee PB, Lee CJ, et al. Dermatome variation of lumbosacral nerve roots in patients with transitional lumbosacral vertebrae. Anesth Analg 2008;106:1279-83 CrossRef Medline 